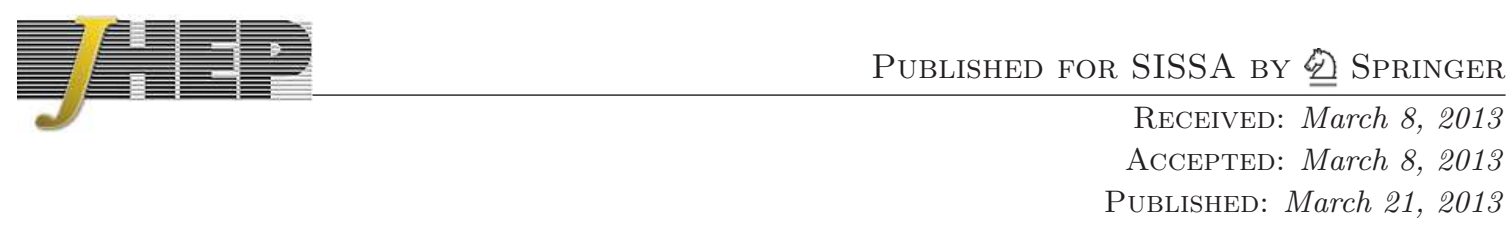

\title{
Erratum: dangerous skyrmions in little Higgs models
}

\section{Marc Gillioz}

Institute for Theoretical Physics, University of Zurich, Winterthurerstrasse 190, CH-8057 Zürich, Switzerland

E-mail: gillioz@physik.uzh.ch

ERRATUM TO: JHEP02(2012)121

ArXiv EPRINT: 1111.2047

There is a confusion in the original paper between the coefficient multiplying the Wess-Zumino-Witten term, eq. (2.2), and the number of colors of the underlying stronglycoupled field theory. The symbol $N_{c}$ is used for both, but only coincide if the technifermions transform in the fundamental representation of the gauge group. Moreover, the coefficient entering eq. (2.2), for which we shall keep the notation $N_{c}$, was claimed to take integer values from topological considerations. However, this depends actually on the form of the matrix $\Phi$. For example, when $\Phi$ transforms in the two-index symmetric representation of $\mathrm{SU}(N)$, as in eq. (3.3), $N_{c}$ can take half-integer values.

The correct relation between the coefficient $N_{c}$ and the true number of colors of the underlying theory, call it $n_{c}$, is dependent on the representation of the technifermions. If the latter are Dirac fermions transforming in the fundamental of a $\mathrm{SU}\left(N_{c}\right)$ gauge group, one has, as mentioned above,

$$
N_{c}=n_{c} \quad \text { (fundamental). }
$$

If on the contrary they are Weyl fermions transforming in the adjoint representation, as in refs. [45-48], one finds

$$
N_{c}=\frac{n_{c}^{2}-1}{2} \quad \text { (adjoint). }
$$

Similar relations can be established for higher-dimensional representations.

The conclusions of the paper remain unchanged. Only the interpretation of the quantity denoted by $N_{c}$ is altered. In terms of the true number of colors $n_{c}$, eq. (3.14) would become for example

$$
q= \begin{cases}\frac{n_{c}^{2}-1}{2} & \text { if } n_{c}^{2}-1 \text { is even (boson), } \\ \frac{n_{c}^{2}-1}{2} \pm 1 & \text { if } n_{c}^{2}-1 \text { is odd (fermion), }\end{cases}
$$

which is anyway incompatible with $q=0$ for $n_{c} \geq 2$. The possible half-integer value of the electric charge for the skyrmion is not in contradiction with the integer electric charge of the Goldstone bosons, since in this case massive fermions must be added to the effective Lagrangian description, as argued in refs. [45-48]. 